\title{
Transparent current spreading layers for optoelectronic devices
}

\author{
A. Porch, D. V. Morgan, and R. M. Perks \\ School of Engineering, Cardiff University, Cardiff, CF24 OYF, United Kingdom \\ M. O. Jones and P. P. Edwards \\ Inorganic Chemistry Laboratory, Oxford University, Oxford, OX1 3QR, United Kingdom
}

(Received 1 March 2004; accepted 30 June 2004)

In this paper we develop a simple model based on a leaky transmission line to assess the electrical performance of transparent current spreading layers for application to surface light emitting diodes. Figures of merit suitable for device design are obtained and these are applied to a range of material systems reported in the literature. These calculations show that materials with electrical characteristics approaching ultimate performance limits can be achieved. Furthermore, calculations of the electromagnetic absorption of conducting layers show that further improvements should be possible by selecting materials with the highest possible majority carrier mobilities, which enable films of greater thickness to be grown while maintaining high transparency, leading to very low spreading layer resistances. Ways of achieving improved mobility are also discussed. (C) 2004 American Institute of Physics. [DOI: 10.1063/1.1786674]

\section{INTRODUCTION}

A critical consideration in the design of semiconductor devices is the requirement to inject current into the structure in an efficient manner. The metallic bond wire (or interconnect) does this through two elements: the ohmic contact and the current spreading layer. This paper deals with the second of these issues, specifically the design of transparent currentspreading layers suitable for the use in optoelectronic devices such as surface emitting light emitting diodes (LEDs) and lasers.

The function of the current spreading layer is to ensure that the injected current is spread as evenly as possible across the whole active "near surface" area of the device. For conventional electronics, this is achieved by the use of appropriate highly conductive metal layers. For optoelectronic devices, such layers present severe limitations due to their lack of transparency to light. Alternative "transparent" conductive layers are necessary if light is to be extracted from the diode. In some cases, heavily doped semiconductors with a band gap $E_{g}$ larger than that of the active junction region of the device have been grown epitaxially onto the semiconductor to form the spreading layer. An example is $p$-doped $\mathrm{GaP}$ grown on AlGaInP device structures. ${ }^{1,2}$ Conductive layers of thicknesses up to $15 \mu \mathrm{m}$ have been grown in this way. When epitaxial growth is not possible, deposited layers of wide band gap materials such as indium tin oxide (ITO) have proved to be very effective alternatives. Some examples ${ }^{3,4}$ of such systems are summarized in Table I. In this paper, consideration is given to the optimum design of these layers for use in optoelectronic devices.

\section{A "FIGURE OF MERIT" FOR DESIGNING LED SPREADING LAYERS}

In order to design optimal transparent spreading layers for surface emitting LEDs, the identification of a figure of merit could be an invaluable aid for the device designer.
Figure 1 illustrates a surface emitting LED typical of most device structures investigated to date. ${ }^{1,2}$ A transparent conducting spreading layer is grown, or deposited, onto the upper semiconductor surface of the LED. In the case of $\mathrm{AlGaInP}$ devices, the inclusion of a spreading layer is essential since the upper surface is normally a low doped $p$-type region. It is low doped owing to the difficulty of incorporating heavy $p$ doping into the layers during growth. A circular metal contact, of radius $r_{0}$, is then placed at the center of the chip, onto which is attached a bond wire. With this simple geometry, the diode current $I_{d}$ is both injected directly across the junction below the contact $\left(I_{c}\right)$ and also spread out radially through the current spreading layer $\left(I_{s}\right)$, as illustrated in Fig. 1, where $I_{d}=I_{c}+I_{s}$. As the current spreads radially, a fraction of $I_{d}$ (i.e., $I_{s}$ ) continually leaks across the $p n$ junction. This, in turn, produces light, part of which is emitted from the diode through the transparent conductor.

The process of current spreading has been modeled using a lossy transmission line model, ${ }^{2}$ as shown in Fig. 1 and Fig. 7 in the Appendix. In such a system, a figure of merit needs to include three elements: (i) the fraction of the diode

TABLE I. Calculated values of $R_{\square}, r_{d}$, and $f$ for a selection of transparent layers of excellent performance reported in the literature (see Ref. 4). These are for indium tin oxide (ITO), indium oxide (IO), cadmium tin oxide (CTO), and indium zinc oxide (IZO). The calculations were carried out for the layer thicknesses listed in the thickness column $(t)$.

\begin{tabular}{cccccc}
\hline \hline Material & $t(\mu \mathrm{m})$ & $R_{\square}(\Omega)$ & $r_{d}(\mu \mathrm{m})$ & $f$ & $T$ (approximately) \\
\hline ITO & 1.0 & 2.0 & 100 & 4.0 & 0.9 \\
IO & 0.5 & 1.5 & 114 & 4.5 & 0.85 \\
CTO & 1.0 & 1.7 & 107 & 4.3 & 0.85 \\
IZO & 0.7 & 11.4 & 42 & 1.7 & 0.9 \\
ITO & 0.7 & 2.5 & 89 & 3.6 & $\cdots$ \\
ITO & 0.07 & 25 & 28 & 1.1 & $\cdots$ \\
& & & $62^{\mathrm{a}}$ & $2.5^{\mathrm{a}}$ & \\
\hline
\end{tabular}

$\overline{\bar{a}}$ Value corrected for diode conductance $G_{d}$ and the low doped $p$-type series resistance. 


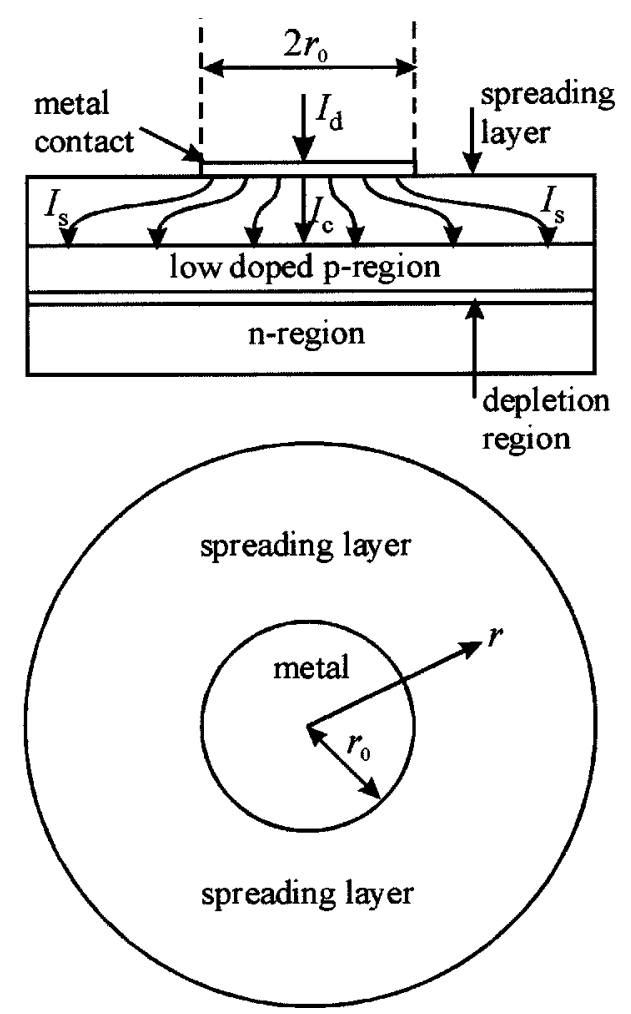

FIG. 1. A schematic diagram of a typical AlGaInP surface light emitting diode. The side view shows the current spreading layer sitting on top of the basic $p n$ junction diode. Also shown are the total current $I_{d}$ and the components $I_{s}$ injected into the spreading layer and $I_{c}$ crossing the junction immediately below the metal contact. The plan view shows a circular metal contact of radius $r_{0}$, along with the radial distance $r$ measured from the center of the contact.

current that is injected into the spreading layer, i.e., $g$ $=I_{s} / I_{d}$ or, equivalently, the fraction of the spreading current to the current injected beneath the contact, i.e., $f=I_{s} / I_{c}$ $=g /(1-g)$, (ii) the effectiveness of the layer in spreading the current radially, and (iii) the transmission coefficient of the spreading layer for emitted photons.

\section{A. Spreading resistance}

Consider the first two of the above elements [i.e., (i) and (ii)]. The Appendix presents a simple analysis of a leaky transmission line model for the case of a circular [i.e., twodimensional (2D)] geometry, as illustrated in Fig. 7 of the Appendix. An even simpler 1D current spreading geometry is also described for a square or rectangular contact. For their current spreading properties, the solutions for both geometries approximate to a similar functional dependence of current falloff with distance from the edge of the metallic contact. This paper will concentrate on the more complex circular geometry since it closely approximates the basic surface emitting LED structures reported to date, ${ }^{1,2}$ though the 1D model is suitable for some laser diode structures.

Equation (A1) in the Appendix gives the approximate radial distribution of the spreading current $I_{s}(r)$ as

$$
I_{s}(r) \propto \sqrt{\frac{G}{R_{\square}}} r K_{1}(\alpha r) \approx \frac{C}{\sqrt{\alpha}} \exp (-\alpha r),
$$

where $K_{1}(x)$ is the first order, modified Bessel function of the second kind, $\alpha=\sqrt{R_{\square} G}$, and $C$ is a constant. Equation (1) is valid in the limit when $\alpha r_{0} \gg 1$ for the region $r \geqslant r_{0}$; for the region defined by $r<r_{0}$ the metal contact is assumed to be an equipotential surface, with constant current density $J_{c}$ leading to an injected current into the junction beneath the contact of $I_{c} \approx \pi r_{0}^{2} J_{c}$.

We define the radial decay length $r_{d} \equiv \alpha^{-1}=\left(R_{\square} G\right)^{-1 / 2}$, which is the radial distance from the contact edge where the current density has fallen to $1 / e$ of its value at the edge of the contact. From Eq. (A2), the spreading resistance for a circular metal contact of radius $r_{0}$ is then approximately

$$
R_{s} \approx \frac{1}{2 \pi r_{0}} \sqrt{\frac{R_{\square}}{G}} .
$$

The equivalent circuit for the whole diode is shown in Fig. 7 of the Appendix, including the two components of currents $I_{c}$ and $I_{s}$ that make up the diode current $I_{d}$. Since $I_{c}$ leads to very little light output since it is associated with light generation beneath the metal contact, a useful figure of merit is therefore the fraction

$$
f \equiv \frac{I_{s}}{I_{c}} \approx \frac{1}{G \pi r_{0}^{2}} \frac{1}{R_{s}} \approx \frac{2}{r_{0}} \frac{1}{\sqrt{R_{\square} G}} \equiv \frac{2 r_{d}}{r_{0}} .
$$

Numerical values of $r_{d}, R_{s}$, and $f$ all provide useful measures of the effectiveness of the current spreading layers, and are interrelated by Eqs. (2) and (3) above. For example, a large value of $r_{d}$ would indicate that the layer is effective in its spreading properties, and can be increased by decreasing the sheet resistance $R_{\square}$ of the spreading layer. The conductance $G$ per unit area is determined by the LED junction resistance at its bias point and is a function of the diode drive current $I_{d}$.

Ignoring for the moment the effects of the series resistance of the $p n$ junction, the diode forward conductance is approximately

$$
G_{d} \approx\left(\frac{q}{k T}\right) I_{d} \equiv G \pi r_{0}^{2}
$$

and the sheet resistance is defined to be $R_{\square}=\rho / t=\left(q n \mu_{e} t\right)^{-1}$. Hence,

$$
r_{d} \equiv \frac{1}{\sqrt{R_{\square} G}} \approx r_{0}\left(\frac{\pi k \operatorname{Tn} \mu_{e} t}{I_{d}}\right)^{1 / 2}
$$

so that for a constant diode current $I_{d}$,

$$
f=\frac{2 r_{d}}{r_{0}} \propto\left(n \mu_{e} t\right)^{1 / 2} .
$$

Equations (4) and (5) show that the effectiveness of the current spreading layer increases as the square root of the free electron density $n$, electron mobility $\mu_{e}$, and layer thickness $t$. These conclusions will be discussed later in the paper. 


\section{B. Transparency of a spreading layer}

A conducting medium is transparent to electromagnetic waves provided that the photon energy does not exceed the band gap of the material. For the transmission of visible light of free space wavelength in the range $390-780 \mathrm{~nm}$, a band gap $E_{g}>3.2 \mathrm{eV}$ is required. Since $E_{g} \approx 3.75 \mathrm{eV}$ for indium tin oxide (ITO), this condition is satisfied for ITO, and will also form the base criterion for alternative contenders for the role of transparent spreading layers. A small selection of some of the best results are summarized in Table I (more extensive listings of results appear in the literature ${ }^{4}$ ).

There are, however, other considerations that determine the ultimate transparency of a spreading layer. These include (i) absorption by free electrons, and (ii) absorption by defects which produce impurity levels within the band gap.

\section{Absorption by free electrons}

A conducting film will be transparent if the frequency $\omega$ of the incident light exceeds the plasma frequency $\omega_{p}$ (assuming also, of course, that $\hbar \omega<E_{g}$ ), but otherwise highly reflecting. In this statement, it is assumed implicitly that the thickness of the film $t$ is less than the electromagnetic skin depth $\delta$ (this point will be reviewed below). It has been shown that the optical properties of ITO are fairly well described by the Drude model of a free electron gas. ${ }^{5,6}$ Using the Drude model it can be shown that a film is nonreflective when the incident wavelength is less than the critical value $\lambda_{p}=2 \pi c / \omega_{p}$, where $\omega_{p}$ is the plasma frequency. Using values from the literature ${ }^{7}$ for ITO, the free electron density $n$ for optical transparency at some incident free space wavelength $\lambda_{0}$ must satisfy the equation

$$
n\left(\mathrm{~cm}^{-3}\right)<1.6 \times 10^{27} / \lambda_{0}^{2}\left(\mathrm{~nm}^{2}\right) .
$$

For efficient transmission of the whole visible spectrum (including red light of wavelength up to $780 \mathrm{~nm}$ ), the free electron density should therefore not exceed 2.6 $\times 10^{21} \mathrm{~cm}^{-3}$. To allow a suitable "safety margin," the plasma wavelength is assumed to be $1 \mu \mathrm{m}$ (i.e., in the near infrared), in which case the maximum tolerable free electron density is $n_{\max } \approx 1.6 \times 10^{21} \mathrm{~cm}^{-3}$.

Published data ${ }^{3,4}$ suggest that this value has already been attained in amorphous ITO, indium oxide (IO), and cadmium tin oxide (CTO) layers deposited on glass. To maximize the dc conductivity $\sigma(0)=n q \mu_{e} \equiv n q^{2} \tau(0) / m^{*}$ of such layers, and hence reduce the dc spreading resistance for LED-based applications, clearly $n$ cannot be further increased without shifting the plasma edge to within the visible portion of the spectrum. Therefore, the issue of how the electron mobility $\mu_{e}=q \tau(0) / m^{*}$ can be further increased in these $n$-type layers needs to be addressed, together with the effects of this increased electron mobility on the optical properties of ITO layers.

Porch et al. ${ }^{7}$ have addressed this issue by calculating the skin depth $\delta$ as a function of mobility and carrier density at frequencies above the plasma edge, the most important result being

$$
\delta(\omega) \approx \frac{2 m^{*}}{Z_{\propto} q^{2}} \frac{\omega^{2} \tau}{n} \propto \frac{\omega^{2} \mu_{e}}{n},
$$

where $Z_{\infty} \approx 179 \Omega$ is the wave impedance of ITO in the limit $\omega \gg \omega_{p}, m^{*} \approx 0.35 m_{e}$ is the effective mass of conduction band electrons, and $\tau$ is their scattering time at optical frequencies. Hence, the simple functional dependences of Eq. (7) show that while increasing $n$ leads to a reduction in the skin depth and an associated reduction in the transparency of the film, an increase in dc mobility actually increases the skin depth and enhances the transparency of the film. This yields the comforting situation that increasing the dc mobility of an ITO film provides superior dc properties (and hence current spreading in LED applications) while also aiding its optical transparency.

To quantify this discussion, assuming that $n \approx 1.6$ $\times 10^{21} \mathrm{~cm}^{-3}$ and $\tau \approx 3.3 \times 10^{-15} \mathrm{~s}$ sets the plasma wavelength $\lambda_{p} \approx 1 \mu \mathrm{m}$ (i.e., in the near infrared) and the dc mobility at $\mu_{e} \approx 50 \mathrm{~cm}^{2} \mathrm{~V}^{-1} \mathrm{~s}^{-1}$ [also assuming that $\tau(0) \approx 3 \tau$ $\approx 10^{-14} \mathrm{~s}$ ]. Transmission of red light at $700 \mathrm{~nm}$ [which will yield a lower limit of the skin depth in the optical spectrum since $\left.\delta(\omega) \propto 1 / \omega^{2}\right]$ has an associated skin depth of $1.4 \mu \mathrm{m}$ calculated using exact analysis, ${ }^{7}$ or about $2.0 \mu \mathrm{m}$ using the approximate analysis [Eq. (7)]. Hence, there will be less than $13 \%$ decrease of the optical power due to electromagnetic absorption in ITO films in the visible spectrum for film thicknesses up to around $100 \mathrm{~nm}$ [i.e., up to about $\delta / 14$, so that the optical power transmitted $T \approx \exp (-2 t / \delta) \approx 87 \%]$. The dc conductivity of the film is $\sigma(0)=n q \mu_{e} \approx 1.3 \times 10^{4} \mathrm{~S} \mathrm{~cm}^{-1}$, so that a film of thickness $t=100 \mathrm{~nm}$ would have a small sheet resistance of $R_{\square}=1 / \sigma(0) t \approx 8 \Omega$, an encouraging value for current spreading layers.

If electron mobilities in excess of $\mu_{e} \approx 50 \mathrm{~cm}^{2} \mathrm{~V}^{-1} \mathrm{~s}^{-1}$ could be achieved while maintaining the high free electron density (but keeping the plasma edge in the infrared) then the benefits for current spreading layers would be twofold: (i) clearly, the dc conductivity would be increased, thus reducing the sheet resistance, but (ii) the skin depth would also be increased, allowing thicker films to be grown, and even further reducing the sheet resistance. For example, exact calculations of the skin depth show that for the red light transmission, if the dc mobility could be increased by a factor of 2 for fixed $n$ then the skin depth increases by the factor 1.97 [simple modeling using Eq. (7) predicts a factor of 2 increase]. One would then expect this factor of 2 increase in the mobility to enable a factor of 4 decrease in the sheet resistance while preserving the same optical properties of the film, a very significant result.

\section{Absorption by defects and impurities}

A second source of photon absorption is impurity energy levels within the band gap arising from crystalline defects and impurity complexes in the films, which lead to photon absorption at energies $<E_{g}$. Such impurity centers have been observed to occur in both sputtered and thermally deposited thin ITO films ${ }^{3}$ and could account for the absorption seen. Absorption gives "as-deposited" films a yellow (sputtered) or gray (evaporated) appearance, and can be partially removed by the same annealing process used to improve the conduc- 


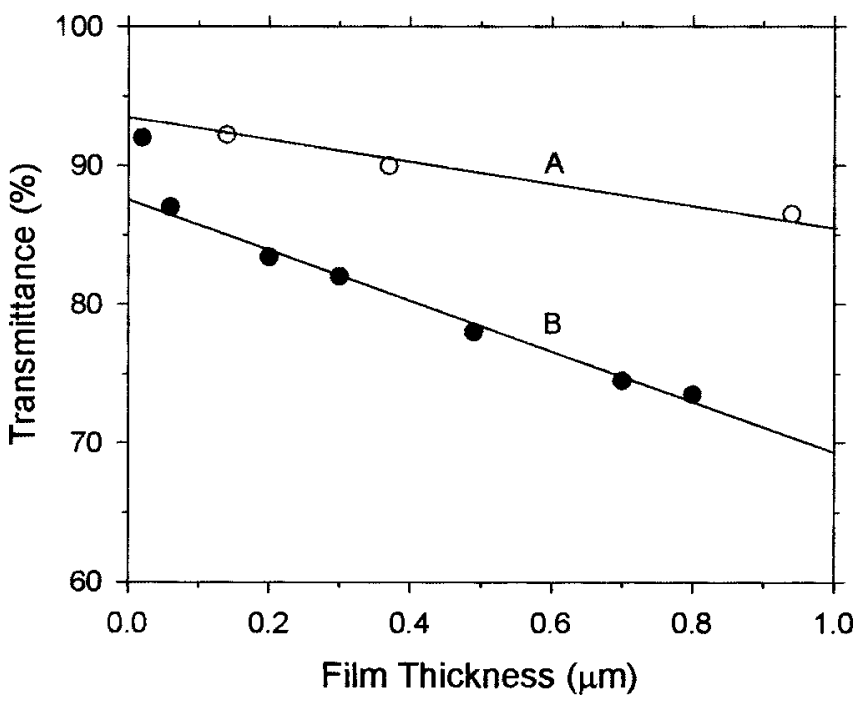

FIG. 2. A plot of the transmission coefficient $T$ as a function of the ITO layer thickness $t$ for two extreme examples: a layer deposited by pyrolitic spray deposition (see Ref. 8) (data set A, open circles) and an unannealed thermal evaporated layer (see Ref. 2) (data set B, closed circles). These are typical of the range of values reported in the literature and indicate that excellent results can be obtained with low sheet resistances (i.e., $R_{\square} \approx 2 \Omega$ ).

tivity and electron mobility of the ITO. The fact that improved transmittance occurs simultaneously with increased free carrier density, coupled with the fact that the improved carrier density $n \approx 10^{21} \mathrm{~cm}^{-3}$ is below the value of 1.6 $\times 10^{21} \mathrm{~cm}^{-3}$ calculated earlier as the critical upper limit [Eq. (6)], would suggest that the major source of absorption in these films is due to defect/impurity levels, rather than from free electrons in the conduction band; this is also in agreement with the discussion of the preceeding section, where it was shown that the optical skin depth in the films was large (typically $>1 \mu \mathrm{m}$ ). The consequence of impurity/defect centers is the unacceptably large value of absorption observed in some sputtered and thermally evaporated layers ${ }^{3}$ of ITO compared with those reported which have been fabricated by other techniques. ${ }^{4,8-11}$

A plot of two reported sets of data is shown in Fig. 2. Up to film thicknesses of around $1 \mu \mathrm{m}$ the transmittance curves can be fitted approximately to a straight line

$$
T(t)=T(0)-\beta t,
$$

where $T(t)$ is the transmittance at depth $t, T(0)$ the corresponding value at the surface, and $\beta$ the absorption coefficient. The values of $\beta$ obtained for the two curves shown in Fig. 2 are $0.19(\mu \mathrm{m})^{-1}$ (thermally evaporated) and $0.08(\mu \mathrm{m})^{-1}$ (spray pyrolysis). The lower experimental figure of about $8 \%$ per micron for the spray pyrolysis technique is an excellent result, but the absorption of the thermally evaporated ITO is too high for practical applications. There is, however, evidence that the annealing of films in an inert atmosphere improves the transmittance. ${ }^{3}$ The thermally evaporated layer in Fig. 2 was deposited on a heated substrate (at $250{ }^{\circ} \mathrm{C}$ ) but had not been subjected to any post deposition annealing. Using a heated substrate improves the transmittance of the layers substantially. Nevertheless, the present result of about $19 \%$ per micron is still too high. The objective must be to reduce absorption to a level of around or

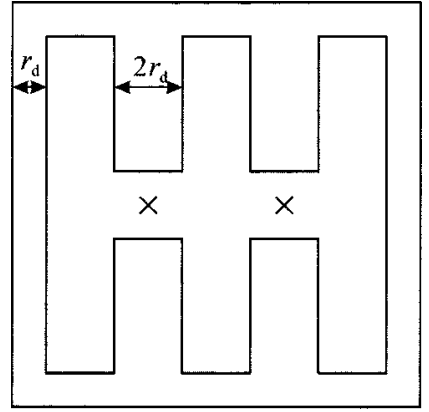

FIG. 3. A schematic of a herring bone geometry device, an alternative to the simple circular geometry used in the basic structures shown in Fig. 1. Reference 1 gives other examples where the ratio of peripheral length to contact area is optimized.

below the figure of $8 \%$ per micron achieved by the best reported techniques. Results from sputtered films ( $\mathrm{dc}$ and magnetron) have already achieved this goal. ${ }^{4,8-11}$ Sputtering and vacuum evaporation are preferred techniques for film deposition because they are compatible with present semiconductor device technology.

\section{MATERIAL AND DEVICE DESIGN CONSIDERATIONS}

\section{A. Maximizing the current spreading}

Equations (3) and (4) provide the figures of merit $f$ and $r_{d}$ for the design of the current spreading layers in a surface emitting LED of circular contact geometry, both of which are maximized by minimizing the sheet resistance $R_{\square}$, i.e., by maximizing the product $\left(n \mu_{e} t\right)^{1 / 2}$. However, as discussed in the preceding section, increasing either $n$ or $t$ can give rise to a reduction in transmittance.

One remaining factor in Eq. (3) that can be varied is the contact radius $r_{0}$. The fraction $f$ is proportional to $r_{0}^{-1}$ and hence increases as $r_{0}$ decreases. This is simply a consequence of increasing the ratio of the contact perimeter $2 \pi r_{0}$ to the contact area $\pi r_{0}^{2}$. As a design parameter, this is very limited for the simple case of a circular contact for two reasons. First, there is the practical issue of the minimum contact area being dictated by the bond wire contact. Second, as the contact radius $r_{0}$ decreases, the total light emitting area (which scales approximately as $r_{0} r_{d}$ ) decreases, which in effect reduces the total optical output power of the diode.

The maximization of $f$ is better achieved by using alternative contact geometries which suit more favorably the criteria of increasing the ratio of the contact perimeter to the contact area without the penalty of a loss of emission area. The review by Kish and Fletcher ${ }^{1}$ discusses alternative contact geometries. Another contender is the "herring bone" geometry shown in Fig. 3. This can also combine a high emitting ratio of peripheral length to contact area. The separation of the spines by a distance $2 r_{d}$ would ensure that the whole chip outside of the metal contact emitted evenly. To achieve the full effect, the metal contact between the bond and the extreme end of a finger must have an excellent (i.e., low value) of sheet resistance to ensure that the metal surface is maintained as an equipotential surface. This is necessary to keep the voltage drop between the contact bond and the ex- 
treme end of the metallization very small (less than about $0.01 \mathrm{~V}$ ), which would ensure that the whole region of the diode is turned on and thus emitting efficiently. The use of a double bond at positions marked by the crosses in Fig. 3 would help in this context.

\section{B. Carrier density and mobility}

The analysis presented in Sec. II B 1 derives an approximate upper limit to the free electron density $(n \approx 1.6$ $\times 10^{21} \mathrm{~cm}^{-3}$ ) to ensure that a conducting film does not become reflecting within the visible portion of the spectrum. Values of this order of magnitude $\left(5 \times 10^{20}\right.$ to 2 $\times 10^{21} \mathrm{~cm}^{-3}$ ) have already been reported ${ }^{3,4,8-11}$ and some of the key achievements are summarized in Table I. ITO, IO, and $\mathrm{CTO},{ }^{3,4,8-10}$ in particular, have been reported to have free electron densities in excess of $10^{21} \mathrm{~cm}^{-3}$. It may therefore be concluded that the potential for further decreases in sheet resistance $R_{\square}$ by increasing $n$ alone is very limited. However, Eq. (7) shows that the absorption of photons by conduction electrons decreases with increasing electron mobility $\mu_{e}$, so that an increase in $\mu_{e}$ would both increase the conductivity directly and aid the transparency of the film; this means that thicker films can be grown, so that the sheet resistance is reduced by a combination of both increased mobility and film thickness. At the present time the reported electron mobility values for ITO range from 10 to $40 \mathrm{~cm}^{2} \mathrm{~V}^{-1} \mathrm{~s}^{-1}$. Morgan et al. ${ }^{3}$ have shown that an increase in mobility correlated closely with an increase in grain size. However, an unresolved issue is by how much the mobility can be increased by annealing. It is of particular concern that any annealing of an LEDs structure must be compatible with interface stability. This point has been emphasised by Morgan, Aliyu, and Bunce, ${ }^{12}$ who reported that ITO-GaAs Schottky contacts became ohmic on annealing. In the present context, however, an improvement in ohmicity of the ITO-semiconductor interface would improve device performance rather than degrade it.

In the context of increased mobility, it is possible that more complex spreading layers might need to be investigated. A recent publication ${ }^{13}$ reports crystalline $\mathrm{InGaO}_{3}(\mathrm{ZnO})$ transparent layers with a mobility of up to $140 \mathrm{~cm}^{2} \mathrm{~V}^{-1} \mathrm{~s}^{-1}$, and one might speculate that potential values up to $200 \mathrm{~cm}^{2} \mathrm{~V}^{-1} \mathrm{~s}^{-1}$ might be achievable. This would be an increase of an order of magnitude over the mobility values reported on polycrystalline layers.

\section{Comparisons with published results}

Table I summarizes a selection of the best results reported in the literature for a range of transparent conductors. ${ }^{3,4}$ Also shown are the sheet resistance values calculated on the basis of the resistivities and film thicknesses reported. These values of $R_{\square}$ confirm that sheet resistances as low as $2 \Omega$ are achievable with ITO, $1.7 \Omega$ for CTO, and $1.5 \Omega$ for IO. In the first two cases the value of $t=1 \mu \mathrm{m}$ and in the last case $t=0.5 \mu \mathrm{m}$ were measured ${ }^{9,10}$ and used to calculate $R_{\square}$. Published ITO data, ${ }^{3,4}$ indicate that optimized layers exhibit excellent values for transmittance (Fig. 2). Both curves shown in this figure converge to $90 \%-93 \%$ transmittance when extrapolated to $t=0$. The corresponding values of transmittance are $89.6 \%$ for a pyrolitic sprayed ITO film of thickness $1 \mu \mathrm{m}$ and $73 \%$ for "as deposited" thermally evaporated ITO film of thickness $0.8 \mu \mathrm{m}$. The result for the pyrolitic film is excellent and at $1 \mu \mathrm{m}$ thickness such a film would prove to be an almost ideal current spreading layer. Thermally evaporated or sputter deposited films have the advantage that their technology is compatible with present semiconductor device technology. It has already been shown that annealing the film to modest temperatures improves their spreading layer characteristics; ${ }^{3}$ that is, it produces a significant increase in free electron density $n$, electron mobility $\mu_{e}$, and transmittance $T$. Therefore, there might be optimism in the view that isochronal annealing can provide a suitable technology for vacuum deposited ITO with performance levels comparable with those of the pyrolitic spray and sputtered films.

Also shown in Table I are the calculations of the figures of merit $r_{d}$ and $f$ for a selection of results reported. In the first three cases the lowest $R_{\square}$ are selected, regardless of the deposition technique used. These "best results" are for ITO,IO, CTO, and IZO and the transmittance data reported indicated that films up to $1 \mu \mathrm{m}$ thick can be deposited with excellent values of transmittance [i.e., very low values of loss $\beta$ in Eq. (8)]. Two other results are also included, both for thermally evaporated layers and the technology used by Morgan Al-Ofi, and Aliyu ${ }^{2}$ for LED device fabrication. The first result cited is for a layer of thickness $0.8 \mu \mathrm{m}$ which had not been annealed after deposition but was deposited onto a substrate held at $250{ }^{\circ} \mathrm{C}$. Such layers exhibited an unacceptably high total film absorption $(\approx 19 \%$ at $0.8 \mu \mathrm{m}$, but a very low sheet resistance of $2.5 \Omega$. The final result in Table I is for a thermally deposited layer of thickness $70 \mathrm{~nm}$, corresponding to the actual layer used in LED fabrication. ${ }^{2}$ This is a very thin layer and consequently, while the total absorption in the film will be low, the corresponding sheet resistance rises to $25 \Omega$.

Thus in the device structure a value of $R_{\square}=25 \Omega$ yields values of $r_{d}=28 \mu \mathrm{m}$ and $f=1$.1. Increasing the ITO thickness to $1 \mu \mathrm{m}$ would result in major improvements in performance; $r_{d}$ would increase to $\approx 100 \mu \mathrm{m}$ and $f$ to around 4 . All the values were calculated for a drive current of $20 \mathrm{~mA}$. It must be remembered, however, that increasing the thickness to $1 \mu \mathrm{m}$ would depend critically on ensuring a "state-of-theart" reduction in film absorption, as discussed earlier.

Consider again the value of $r_{d}=28 \mu \mathrm{m}$ for the actual device structure fabricated by Morgan Al-Ofi, and Aliyu, ${ }^{2}$ this would appear to be under half the value observed experimentally (i.e., shown in Fig. 4, which yields $\approx 74 \mu \mathrm{m}$ ). The calculation of $G_{d}$, however, ignores the series resistance of the relatively low doped $p$-type cladding region, which is in series with the junction depletion resistance. Its effect would be to decrease $G_{d}$ and, consequently, to decrease the effective conductance per unit area $G$. Using the actual device values for the resistivities and thickness of these undepleted semiconductor layers (Morgan, Al-Ofi, and $\mathrm{Aliyu}^{2}$ ), the series resistance reduces the value of $G$ from $5.72 \times 10^{-5}$ to 1.06 


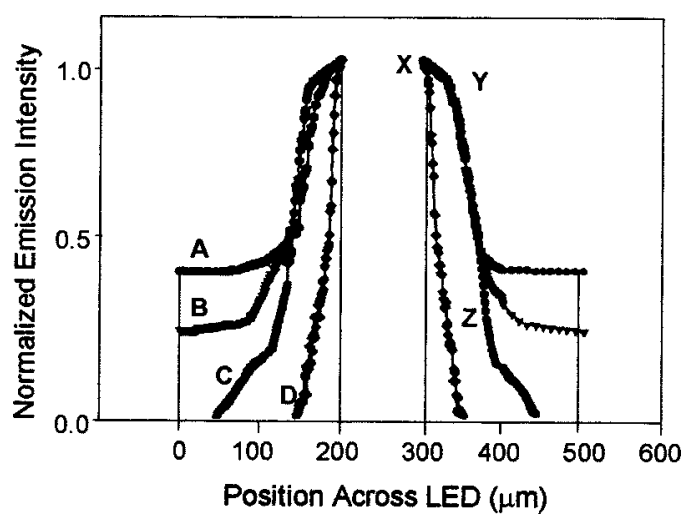

FIG. 4. Plots of light emission as a function of radial distance $r$ obtained by photodensitometer scans across the negatives of the device emission photographs for a range of different LED chips (see Ref. 2). The curves correspond to (a) GaP current spreading layer (CSL), (b) GaInP CSL plus ITO, (c) ITO only CSL, and (d) no CSL.

$\times 10^{-5} \mathrm{~S} \mu \mathrm{m}^{-2}$. This, in turn, increases the calculated value of $r_{d}$ from 28 to $62 \mu \mathrm{m}$, which compares favorably with the experimental value ${ }^{2}$ of around $74 \mu \mathrm{m}$.

Some caution needs to be exercised in estimating the value of $r_{d}$ from the experimental profiles reported in the literature. 1,2,14 For the data of Morgan, Al-Ofi, and Aliyu ${ }^{2}$ (Fig. 4), the profiles were obtained by densitometer scans across photographic negatives. In the case of LEDs with relatively poor spreading layers [curve D of Fig. 4], the profile shows the exponential falloff with distance from the contact edge predicted by Eq. (1). In this case $r_{d}$ can be easily measured. As the sheet resistance $R_{\square}$ decreases then two difficulties arise. The first of these arises due to the limitations of the photographic film used and is exhibited by the three profiles shown in curves $\mathrm{A}$ to $\mathrm{C}$ of Fig. 4. The region $Y Z$ shows the strong predicted exponential decay. However, region $X Y$ exhibits a slow saturation effect due to the saturation of the emulsion in the photographic film. It is in this central region near to the metal contact that the brightness of the diode is highest and its true peak value cannot be estimated. All the data shown in Fig. 4 are normalized at this point and therefore the true form of the radial decay is hidden by the limitation in the experimental procedure. The consequence of this is that it is not possible to estimate $r_{d}$ with any degree of confidence and therefore to test experimentally the relationship in Eq. (1). A second problem arises from the finite size of the LED chip. The analysis presented in Sec. II A assumes that the radial current spreading occurs in an infinite transmission line. When the sheet resistance $R_{\square}$ is high then this indeed is a good approximation and the expected exponential decay is observed. ${ }^{1,2,14}$ As $R_{\square}$ decreases, however, $r_{d}$ begins to approach the chip edge which restricts the outward movement of electrons. The consequence of the finite chip size is that initially a shoulder appears on the photon radial distribution, which in the case of very low values of $R_{\square}$ results in a constant intensity, as is clearly observed in some published data. ${ }^{2,14}$ In the latter case, where the GaP spreading layer is $15 \mu \mathrm{m}$ thick, the whole chip is approaching a constant light output. While this is very desirable from a practical LED point of view, it corresponds to a regime where the transmission model presented earlier is inoperative. The profiles re-

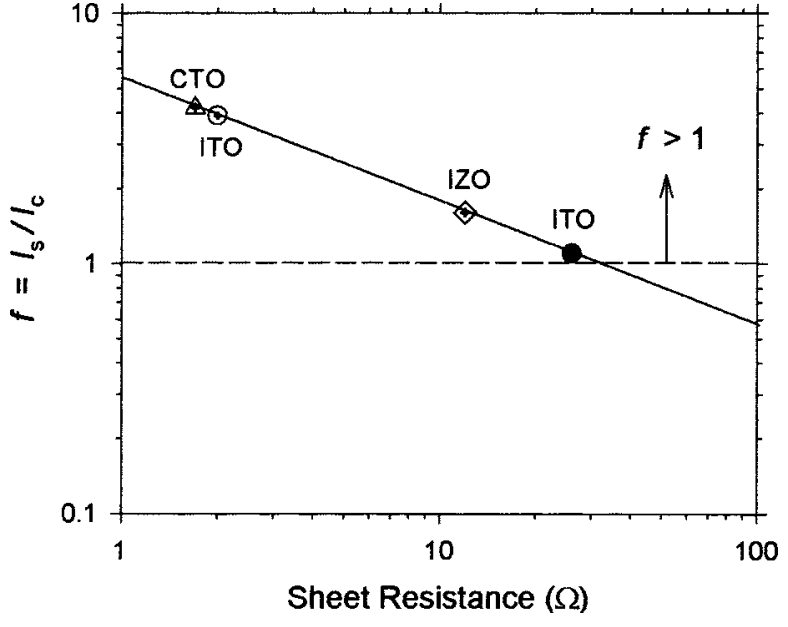

FIG. 5. A theoretical plot of the ratio $f$ of the current injected into the spreading layer current $I_{s}$ to the current injected directly across the $p n$ junction below the metal contact $I_{c}$ [Eq. (9)]. The points on the theoretical curves would be the values appropriate to published values of sheet resistance values $R_{\square}$ reported for CTO, ITO, and IZO. Also shown (O) is the value actually of an ITO layer of a LED structure (see Ref. 2), scaled up to a thickness of $0.7 \mu \mathrm{m}$.

ported by Fletcher et al. ${ }^{14}$ were obtained using a microscope fitted with a video camera and a video analyzer was used to make the measurement. It is not known whether the region near to the contact on the $15 \mu \mathrm{m}$ thick spreading layer was exhibiting a similar saturation effect as that shown in Fig. 4.

Using the LED data of Morgan, Al-Ofi, and Aliyu ${ }^{2}$ (contact radius $r_{0}=50 \mu \mathrm{m}$, diode drive current $I_{d}=20 \mathrm{~mA}$, and $G_{d}=0.4 \mathrm{~S}$ ) then we may write Eq. (3) as

$$
f=\frac{2}{r_{0} \sqrt{R_{\square} G}}=2 \sqrt{\frac{\pi}{G_{d} R_{\square}}} \approx \frac{5.6}{\sqrt{R_{\square}(\Omega)}} .
$$

This relationship is shown logarithmically in Fig. 5. Also shown on this figure are the points corresponding to the bestvalue data used for Table I The ITO, IZO, and CTO data give values of $f$ in excess of 4 , which is a clear indication of their excellent current spreading performance. Figure 5 also shows the corresponding values for the actual ITO layer $(70 \mathrm{~nm}$ thick) used in the devices of Morgan, Al-Ofi, and Aliyu ${ }^{2}$ Here, $f$ still has an acceptable value of 1.1, indicating that the total diode current $I_{d}$ is almost equally shared by $I_{s}$ and $I_{c}$. However, it should be noted that using the corrected value of diode conductance, taking into account the LED series resistance of the low doped $p$ region, brings the value of $f$ up to 2.5.

We can use the same basic diode data to show that

$$
r_{d} \approx \frac{140}{\sqrt{R_{\square}}} \mu \mathrm{m}
$$

so that, in terms of the distance $r$ measured in units of micron from the center of the contact, the following approximation holds for the spreading current 


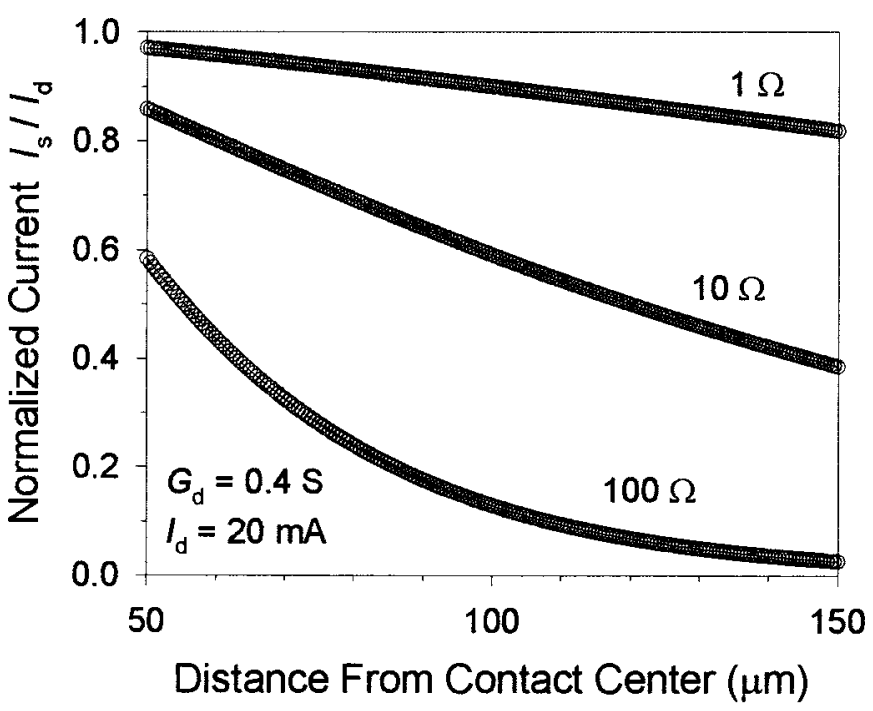

FIG. 6. The spreading current $I_{s}$ normalized to the total diode current $I_{d}$ as a function of the radial distance from the contact center calculated using Eq. (10) for a contact radius of $50 \mu \mathrm{m}$ for three values of spreading layer sheet resistance (i.e., 1, 10, and $100 \Omega$ ).

$$
I_{s}(r) \propto \exp \left(-\frac{r \sqrt{R_{\square}}}{140}\right) .
$$

Figure 6 shows a series of plots of $I_{s}(r)$ versus $r$ for a range of sheet resistances $R_{\square}$ from 1 to $100 \Omega$ for a chip of area $400 \times 400 \mu \mathrm{m}^{2}$ (as reported by Morgan, Al-Ofi, and Aliyu ${ }^{2}$ ). This shows that the current spreading reaches across the whole chip when $R_{\square}<10 \Omega$ and indicates clearly that with present technology, with reported values of $R_{\square}$ as low as $1.5 \Omega$ for a $1 \mu \mathrm{m}$ thick layer, excellent spreading layers can be fabricated which are compatible with semiconductor device technology.

Earlier in this paper it was noted that layers with doping densities near the limit of $1.6 \times 10^{21} \mathrm{~cm}^{-3}$ have already been reported for ITO, IO, and CTO. If the present mobility values of up to around $40 \mathrm{~cm}^{2} \mathrm{~V}^{-1} \mathrm{~s}^{-1}$ can be raised up to around $100 \mathrm{~cm}^{2} \mathrm{~V}^{-1} \mathrm{~s}^{-1}$ by heat treatment, by using more crystalline structures, or by using new materials systems ${ }^{13}$ then values of $R_{\square}<1 \Omega$ could result. This is clearly an achievable goal for optoelectronic device applications.

\section{CONCLUSIONS}

In this paper a simple model based on a circular leaky transmission line is developed for the performance of current spreading layers. The model is applied to conductive transparent spreading layers of the type used for optoelectronic devices. Figures of merit based on the approximate exponential decay length $r_{d}$ and the fraction of total current $f$ injected radially into the spreading layer are proposed as a basis for evaluating potential material systems. The predictions of the equations derived here are compared with the experimental results obtained in the authors' previous studies and with those obtained from the literature.

From these results, it may be concluded that current spreading layers with electrical characteristics approaching their performance limit can be achieved (i.e., free electron densities near to their limit of $2 \times 10^{21} \mathrm{~cm}^{-3}$ have been re-
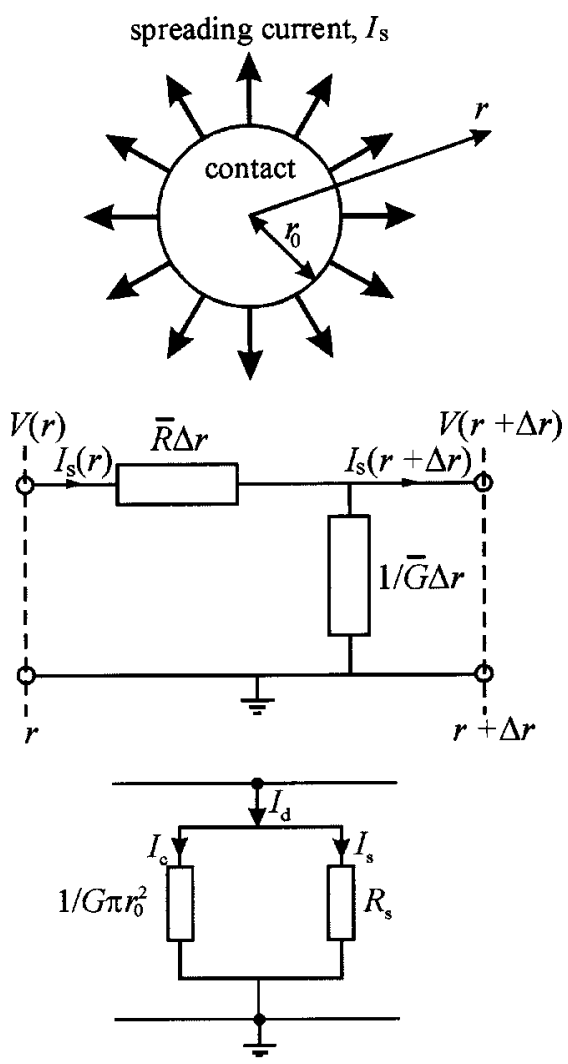

FIG. 7. Details of the geometry of the 2D transmission line model: the device structure, the transmission line model, and the final circuit for the LED.

ported). This paper shows that further improvements will hinge upon increasing the electron mobility $\mu_{e}$. Increases in mobility contribute directly to increased conductivity and reduced sheet resistance.

With regard to film transmittance $T$, some deposition techniques are already capable of producing ITO layers of almost ideal performance with absorption as low as $8 \%$ for $1 \mu \mathrm{m}$ thick films. An objective must now be to obtain these low values with vacuum deposited layers compatible with semiconductor device technology.

\section{APPENDIX: LOSSY TRANSMISSION LINE MODELS FOR CURRENT SPREADING LAYERS}

Figure 7 shows a simplified 2D model of a circular current spreading layer with its lossy transmission line equivalent circuit. We take a annular unit cell of width $\Delta r$ positioned at some radial distance $r\left(\geqslant r_{0}\right)$ from the center of the circular electrode (of radius $r_{0}$ ). We assume that $\bar{R}$ is the resistance per unit length of the spreading layer and $\bar{G}$ is the conductance per unit length of the diode of the LED underneath it. Defining $t, \rho$, and $R_{\square}=\rho / t$ to be the thickness, resistivity and sheet resistance of the spreading layer, respectively, and $G=d I / d V$ the conductance per unit area of the diode, we may write $\bar{R}=R_{\square} / 2 \pi r$ and $\bar{G}=2 \pi r G$. For simplicity we assume that $G$ is independent of voltage above the knee voltage $V_{0}$ of the diode, in which case circuit analysis of the unit cell yields for the voltage $V(r)$, 


$$
\frac{d^{2} V}{d r^{2}}+\frac{1}{r} \frac{d V}{d r}-\alpha^{2}\left(V-V_{0}\right)=0 \quad\left(\alpha^{2}=R_{\square} G\right),
$$

which has solutions $V(r)=V_{0}+A K_{0}(\alpha r)+B I_{0}(\alpha r)$, where $I_{0}(x)$ and $K_{0}(x)$ are zeroth order, modified Bessel functions of the first and second kinds, respectively ( $A$ and $B$ are constants). For a spreading layer of radius $R \gg r_{0}$ we require $B$ $\approx 0$, so the spreading current $I_{s}(r)$ is

$$
I_{s}(r)=-\frac{2 \pi r}{R_{\square}} \frac{d V}{d r} \approx 2 \pi A r \sqrt{\frac{G}{R_{\square}}} K_{1}(\alpha r),
$$

where $K_{1}(x)$ is the first order, modified Bessel function of the second kind. The spreading resistance $R_{S}$ is then

$$
R_{s}=\frac{V\left(r_{0}\right)}{I_{s}\left(r_{0}\right)} \approx \frac{1}{2 \pi r_{0}} \sqrt{\frac{R_{\square}}{G}} \frac{K_{0}\left(\alpha r_{0}\right)}{K_{1}\left(\alpha r_{0}\right)}
$$

in terms of which the current spreading factors $f$ and $g$, and the constant $A$ are

$$
\begin{gathered}
f=\frac{I_{s}(0)}{I_{c}} \approx \frac{1}{\pi r_{0}^{2} G R_{s}}, g=\frac{I_{s}(0)}{I_{d}} \approx \frac{1}{1+\pi r_{0}^{2} G R_{s}}, \\
A=g I_{d} \approx \frac{I_{d}}{1+\pi r_{0}^{2} G R_{s}} .
\end{gathered}
$$

In the limit when $\alpha r_{0} \gg 1$ we find that

$$
\begin{gathered}
I_{s}(r) \propto \exp (-\alpha r), \quad R_{s} \approx \frac{1}{2 \pi r_{0}} \sqrt{\frac{R_{\square}}{G}}, \\
f \approx \frac{2}{r_{0} \sqrt{R_{\square} G}} \propto \sqrt{t} .
\end{gathered}
$$

Identical 1D modeling of a square contact of side $W$ with two adjacent spreading layers of length $L \gg W$ (as appropriate for the geometries of some laser diodes) yields the following very similar results (valid for all $\alpha W$ ):

$$
I_{s}(x) \propto \exp (-\alpha x), R_{s} \approx \frac{1}{2 W} \sqrt{\frac{R_{\square}}{G}}, f \approx \frac{2}{W \sqrt{R_{\square} G}} \propto \sqrt{t},
$$

where $x$ is the distance from the contact center.

The validity of the approximation $\alpha r_{0} \gg 1$ for $2 \mathrm{D}$ current spreading depends on the properties of the both the diode and spreading resistance. The diode conductance is $G_{d}$ $\equiv G \pi r_{0}^{2} \approx q I_{d} / m k T$, so that $\alpha r_{0}=\sqrt{R_{\square} G} r_{0} \equiv \sqrt{R_{\square} G_{d} / \pi}$. Assuming that $m=2$ and $I_{d}=20 \mathrm{~mA}$ (typical of a practical LED application) gives $G_{d} \approx 0.4 \mathrm{~S}$. There are only minor differences in the spatial dependence of the spreading current in the 1D and 2D contact geometries for values of $\alpha r_{0}<1$; when $\alpha r_{0} \gg 1$ both give rise to the same exponential falloff with distance from the contact edge.

${ }^{1}$ F. A. Kish and R. M. Fletcher, High Brightness LEDs, edited by M. G. Craford (Academic, New York, 1997), Chap. 5.

${ }^{2}$ D. V. Morgan, I. M. Al-Ofi, and Y. H. Aliyu, Semicond. Sci. Technol. 15, 62 (2000).

${ }^{3}$ D. V. Morgan, Y. H. Aliyu, R. W. Bunce, and A. Salehi, Thin Solid Films 312, 268 (1998).

${ }^{4}$ K. I. Chopra, S. Major, and D. K. Pandya, Thin Solid Films 102, 1 (1983).

${ }^{5}$ I. Hamberg and C. G. Granqvist, J. Appl. Phys. 60, R123 (1986).

${ }^{6}$ J. R. Bellingham, W. A. Phillips, and C. J. Adkins, J. Mater. Sci. Lett. 11, 263 (1992).

${ }^{7}$ A. Porch, D. V. Morgan, R. M. Perks, M. O. Jones, and P. P. Edwards, J. Appl. Phys. 95, 4734 (2004); P. P. Edwards, A. Porch, M. O. Jones, D. V. Morgan, and R. M. Perks, Dalton Transmissions, B408864F (2004).

${ }^{8}$ R. Pommier, C. Gril, and J. Marucchi, Thin Solid Films 77, 91 (1981).

${ }^{9}$ J. C. C.Fan, F. J. Bechner, and G. H. Foley, Appl. Phys. Lett. 31, 773 (1977).

${ }^{10}$ M. Buchanan, J. B. Webb, and D. F. Williams, Appl. Phys. Lett. 37, 213 (1980).

${ }^{11}$ G. Haacke, Appl. Phys. Lett. 28, 622 (1976).

${ }^{12}$ D. V. Morgan, Y. Aliyu, and R. W. Bunce, Phys. Status Solidi A 133, 77 (1992).

${ }^{13}$ K. Nomura, H. Ohta, K. Ueda, T. Kamiya, M. Hirano, and H. Hosono, Science 300, 1269 (2003).

${ }^{14}$ R. M. Fletcher, C. P. Kuo, T. D. Osentowski, K. H. Huang, M. G. Crawford, and V. M. Robbins, J. Electron. Mater. 20, 1125 (1991). 